\title{
Real-time safety surveillance of seasonal influenza vaccines in children, Australia, 2015
}

\author{
A Pillsbury ${ }^{1}$, P Cashman ${ }^{23}$, A Leeb 4 , A Regan 56 , D Westphal 578 , T Snelling 79 , C Blyth 7910 , N Crawford ${ }^{11213}$, N Wood ${ }^{114}$, K \\ Macartney ${ }^{1215}$, on behalf of the AusVaxSafety, surveillance team ${ }^{16}$ \\ 1. National Centre for Immunisation Research and Surveillance, The Children's Hospital at Westmead, NSW, Australia \\ 2. Hunter New England Local Health District, NSW, Australia \\ 3. The University of Newcastle, NSW, Australia \\ 4. Illawarra Medical Centre, WA, Australia \\ 5. Communicable Disease Control Directorate, Western Australia Department of Health, WA, Australia \\ 6. School of Pathology and Laboratory Medicine, University of Western Australia, WA, Australia \\ 7. Wesfarmers Centre of Vaccines and Infectious Diseases, Telethon Kids Institute, University of Western Australia, WA, Australia \\ 8. National Centre for Epidemiology and Population Health, Research School of Population Health, The Australian National \\ University, ACT, Australia \\ 9. Princess Margaret Hospital, WA, Australia \\ 10. University of Western Australia School of Paediatrics and Child Health, Princess Margaret Hospital, WA, Australia \\ 11. Department of General Medicine, Royal Children's Hospital, Victoria, Australia \\ 12. SAEFVIC, Murdoch Childrens Research Institute, Victoria, Australia \\ 13. Department of Paediatrics, The University of Melbourne, Victoria, Australia \\ 14. Discipline of Paediatrics and Child Health, University of Sydney, NSW, Australia \\ 15.Department of Microbiology and Infectious Diseases, The Children's Hospital at Westmead, NSW, Australia \\ 16. The members of the group are listed at the end of the article.
}

Correspondence: Alexis Pillsbury (alexis.pillsbury@health.nsw.gov.au)

Citation style for this article:

Pillsbury A, Cashman P, Leeb A, Regan A, Westphal D, Snelling T, Blyth C, Crawford N, Wood N, Macartney K. Real-time safety surveillance of seasonal influenza vaccines in children, Australia, 2015. Euro Surveill. 2015;20(43):pii=30050. DOI: http://dx.doi.org/10.2807/1560-7917. ES.2015.20.43.30050 2015;20(34):pii=30002. DOI: http://dx.doi.org/10.2807/1560-7917.ES.2015.20.34.30002

Article submitted on 14 October 2015 / accepted on 29 October 2015 / published on 29 October 2015

Increased febrile reactions in Australian children from one influenza vaccine brand in 2010 diminished confidence in influenza immunisation, highlighting the need for improved vaccine safety surveillance. AusVaxSafety, a national vaccine safety surveillance system collected adverse events in young children for 2015 influenza vaccine brands in real time through parent/carer reports via SMS/email. Weekly cumulative data on 3,340 children demonstrated low rates of fever $(4.4 \%)$ and medical attendance (1.1\%). Fever was more frequent with concomitant vaccination.

In 2014, a multi-jurisdictional national system, AusVaxSafety, was established to undertake enhanced influenza vaccine safety surveillance and report realtime adverse events in children aged six months to four years. This collaborative system was funded by the Australian Government Department of Health. Surveillance $(n=782$ children) demonstrated the safety of 2014 seasonal influenza vaccines in a matter of weeks, although most children received one vaccine brand (Vaxigrip, Sanofi Pasteur; 86.2\%; $n=674$ children) $[1,2]$. Expansion of the programme in 2015 to incorporate a new data management platform and more participating general practice (GP) sites (GPs providemore than $70 \%$ of vaccines given nationally [3]) has enabled reporting of the safety of 2015 southern hemisphere trivalent influenza vaccines for thousands of children receiving multiple manufacturers' vaccines. Here we report the results of our surveillance conducted during the 2015 Australian influenza season.

\section{The AusVaxSafety vaccine safety surveillance system}

In Australia (population 23 million [4]), influenza vaccination is funded under the National Immunisation Program for children aged six months to four years who have medical conditions pre-disposing them to complications and/or for Indigenous children. Only one state, Western Australia (WA), has funded influenza vaccination for this age group since 2008 .

For the purposes of AusVaxSafety surveillance, children aged six months to four years receiving seasonal influenza vaccine from participating GP sites $(n=54)$, hospitals $(n=6)$, public clinics $(n=2)$ and primary healthcare providers such as Aboriginal Medical Services $(n=7)$ in four states (New South Wales (NSW), Victoria, South Australia and WA) were eligible for inclusion. Parent/ carer-reported adverse events in children were solicited within three days of vaccination using two computerbased data management platforms, Vaxtracker [5] and SmartVax [6]. Both systems sent automated SMS messages (and/or emails for Vaxtracker) and received parent/carer-completed questionnaire responses via reply SMS with a URL link to smartphone survey (SmartVax) 


\section{FIGURE}

AusVaxSafety participants with and without postvaccine reaction, by week of vaccination, and cumulative percentage of participants, Australia, 1 April-31 August $2015(n=3,340)$

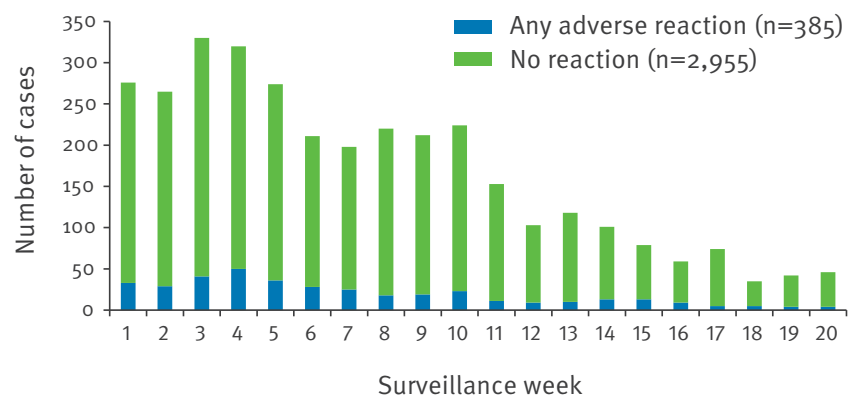

Surveillance Week 1 included all participants vaccinated prior to the official rollout of the influenza vaccine for the 2015 season (20 April 2015) and captured children vaccinated from 1 to 19 April 2015. After that, each surveillance week consisted of seven days, with Week 2 including 20-26 April, etc. Week 20 included eight days (24-31 August 2015).

or web-based survey (Vaxtracker). Demographic details were obtained, as well as information regarding vaccine brand, medical conditions, concomitant vaccines, reactions and healthcare consultations required after vaccination (including follow-up visit to a GP, emergency department (ED) or hospitalisation).

Serious adverse events (SAE) were categorised according to predefined criteria, which included any untoward medical event that resulted in death, was life-threatening or required hospitalisation [7]. We also included seizures requiring medical attendance (ED and/or hospitalisation) as medically important events. SAEs were reported to state/territory health departments and the Therapeutic Goods Administration as required by legislation. For this report, data were compiled from 1 April through 31 August 2015 and cumulative data reported to health authorities weekly. After week 4 of surveillance, progressive results were periodically made publically available online and shared via immunisation provider networks.

For rapid signal detection, fast initial response cumulative summation (FIR CUSUM) and Bayesian methods $[8,9]$ were employed weekly to estimate the probability that any potential safety signal was true or false based on predetermined expected and threshold rates of two objective outcome measures (fever and medical advice/attendance sought) in relation to the number of reports received. Expected and threshold rates were set according to previous surveillance results and published studies. For fever, the expected rate was $6 \%$ and the threshold rate for triggering a signal was $13 \%$ [5,10-12].

\section{Results}

Approximately $75 \%$ of the 4,441 parents/carers invited agreed to participate, resulting in 3,340 post-vaccination reports (Figure). The majority of parent/carers responded within two hours of being queried. Descriptive details of participants are presented in Table 1.

Weekly analysis using FIR CUSUM and Bayesian methods (conducted 1 April through 5 July 2015) did not demonstrate a safety signal at any time. After the third week of surveillance ( $n=877$ cumulative reports), fever rates remained less frequent than $5 \%$ each week and medical advice/attendance rates remained lower than $2 \%$.

Parent/carer-reported fever was recorded by $4.4 \%$ $(n=148)$; medical advice/attendance was sought by $1.1 \%(n=35)$. Details on reactions and medical advice/ attendance sought are included in Table 2.

Of the 35 children who received medical advice/attendance, 23 reported fever. Five children experienced seizures, four of whom had a history of seizures (three: underlying neurological conditions; one: previous febrile seizures). The fifth seizure case occurred in a child diagnosed with a febrile viral illness. Only three of the children with seizures sought medical attendance and were thus classified as having SAEs; all attended an ED only. One additional SAE was recorded in a child hospitalised with an influenza-like illness and fever. Two of the four children experiencing an SAE had received Vaxigrip, one had received Fluarix and the other received Influvac. All reported improvement within days.

No significant difference was identified between children who had received one of the two most commonly used vaccine brands, Vaxigrip or Fluarix, and who experienced fever or sought medical advice/ attendance. All other brands had been administered in insufficient numbers to reliably report on differences (Table 3). Children receiving other vaccines concomitantly were significantly more likely to experience fever $(60 / 687 ; 8.7 \%)$ than those who did not $(87 / 2,618 ; 3.3 \%)(p=0.000)$. There was no difference between children with and without an underlying condition regarding fever $(29 / 400$ (7.3\%) vs 56/721 (7.8\%)) or medical advice/attendance sought ( $9 / 400(2.3 \%)$ vs $17 / 721(2.4 \%))$.

\section{Discussion}

Our novel system of active, prospective vaccine safety surveillance, AusVaxSafety, has demonstrated in real time that 2015 southern hemisphere influenza vaccines registered for use in young Australian children were safe and well-tolerated. Adverse event rates reported by parents/carers remained low and within expected ranges throughout the surveillance period. The fever rate was lower than the pooled estimate $(6.7 \%)$ in a recent systematic review of randomised control trials of children aged six to 35 months receiving the first dose of a trivalent influenza [12]. 
TABLE 1

Demographic details of AusVaxSafety participants, Australia, 1 April-31 August 2015 ( $\mathrm{n}=3,340)$

\begin{tabular}{|c|c|c|c|}
\hline Variable & Response & Number & Percentage \\
\hline Median age (range) & \multicolumn{3}{|c|}{23.0 months $(6.0-59.9)$} \\
\hline Sex ${ }^{a}$ & Male & $1,781 / 3,314$ & $53.7 \%$ \\
\hline Ethnicity ${ }^{b}$ & Indigenous & $119 / 2,519$ & $4.7 \%$ \\
\hline Underlying medical condition ${ }^{c}$ & Yes & $400 / 1,121$ & $35.7 \%$ \\
\hline Concomitant vaccine(s) received ${ }^{d}$ & Yes & $687 / 3,305$ & $20.8 \%$ \\
\hline
\end{tabular}

a Sex unknown for 26 of 3,340 participants.

${ }^{b}$ Ethnicity unknown for 821 of 3,340 participants.

c Underlying medical condition not available for 2,219 of 3,340 participants (SmartVax data management system does not currently collect this variable).

${ }^{d}$ Data on whether concomitant vaccine was received unknown for 35 of 3,340 participants.

\section{TABLE 2}

Adverse events reported by 2015 AusVaxSafety participants within three days of vaccination, Australia, 1 April-31 August $2015(\mathrm{n}=3,340)$

\begin{tabular}{|c|c|c|c|}
\hline \multicolumn{2}{|l|}{ Adverse event } & Number & Percentage \\
\hline \multicolumn{2}{|l|}{ Any adverse event } & $385 / 3,340$ & $11.5 \%$ \\
\hline \multicolumn{2}{|l|}{ Fever } & $148 / 3,340$ & $4.4 \%$ \\
\hline \multicolumn{2}{|l|}{ Injection site reaction } & $67 / 3,340$ & $2.0 \%$ \\
\hline \multicolumn{2}{|l|}{ Vomiting/abdominal pain } & $41 / 3,340$ & $1.2 \%$ \\
\hline \multicolumn{2}{|l|}{ Rash } & $36 / 3,340$ & $1.1 \%$ \\
\hline \multicolumn{2}{|c|}{ Participants who sought any medical advice and/or required any medical attendance } & $35 / 3,340$ & $1.1 \%$ \\
\hline \multirow{4}{*}{$\begin{array}{l}\text { Highest medical advice and/or } \\
\text { attendance reported }\end{array}$} & $\begin{array}{l}\text { Participants attending a medical facility for consultation with a } \\
\text { general practitioner or other medical practitioner }\end{array}$ & $23 / 3,340$ & $0.7 \%$ \\
\hline & $\begin{array}{l}\text { Participants telephoning a medical facility or a medically staffed } \\
\text { helpline for advice }\end{array}$ & $4 / 3,340$ & $0.1 \%$ \\
\hline & Participants presenting to an emergency department (not admitted) a & $6 / 3,340$ & $0.2 \%$ \\
\hline & Participants hospitalised ${ }^{\mathrm{b}}$ & $2 / 3,340$ & $0.1 \%$ \\
\hline
\end{tabular}

a Of the five children with seizures reported, three presented to an emergency department and were thus classified as having a serious adverse event.

${ }^{b}$ One child was hospitalised with an unrelated condition not deemed a serious adverse event. The other hospitalised child had an influenzalike illness.

Active, prospective vaccine safety surveillance is superior to traditional post-marketing vaccine safety surveillance which typically relies on passive reporting. In Australia, SMS technology has also been used to study vaccine reactions among healthcare workers and pregnant women $[13,14]$. One study in the United States also used SMS follow-up of parents, detecting increased fever rates in children who had concomitantly received trivalent influenza vaccine and 13 -valent pneumococcal vaccine compared with those who received each vaccine alone [15]. Similarly, we reported an increased (although low) rate of fever when influenza vaccine was administered together with other vaccines. This was also associated with a significantly higher likelihood of seeking medical advice and warrants further investigation.
Because large volumes of influenza vaccine are distributed annually within short, defined periods, active surveillance provides the opportunity to gain early, reliable assessments of the safety profiles of new vaccines. As the number of available influenza vaccines increases, obtaining timely safety data becomes more important, particularly as strain composition may vary from season to season. In 2010 in Australia, an unexpected increase in febrile reactions following receipt of influenza vaccination in young children led to a three month suspension of all national paediatric influenza immunisation programmes [16]. Epidemiological and laboratory studies linked these reactions to one manufacturer's vaccine (Fluvax or Afluria, bioCSL) which is no longer registered for use in young children $[16,17]$; however, confidence in all influenza vaccines was negatively impacted $[18,19]$. In response to these safety concerns which have resulted in low uptake of 
TABLE 3

Details of influenza vaccines administered to AusVaxSafety participants, Australia, 1 April-31 August 2015 (n = 3,340)

\begin{tabular}{|c|c|c|c|c|c|c|c|}
\hline \multirow[t]{2}{*}{$\begin{array}{l}\text { Branda } \\
\text { (manufacturer) }\end{array}$} & \multirow[t]{2}{*}{ Vaccine type } & \multicolumn{2}{|c|}{$\begin{array}{l}\text { Number of vaccines } \\
\text { administered } \\
(n=3,336)\end{array}$} & \multicolumn{2}{|c|}{$\begin{array}{c}\text { Number of participants } \\
\text { with } \\
\text { fever by brand }\end{array}$} & \multicolumn{2}{|c|}{$\begin{array}{l}\text { Number of participants who sought } \\
\text { medical advice/attendance by brand }\end{array}$} \\
\hline & & $n$ & $\%$ & $\mathrm{n} / \mathrm{N}$ & $\%$ & $\mathrm{n} / \mathrm{N}$ & $\%$ \\
\hline $\begin{array}{l}\text { Vaxigrip } \\
\text { (Sanofi-Pasteur) }\end{array}$ & Trivalent & 3,075 & 92.2 & $133 / 3,075^{c}$ & $4.3 \%$ & $28 / 3,075^{d}$ & $0.9 \%$ \\
\hline $\begin{array}{l}\text { Fluarix } \\
\text { (GlaxoSmithKline) }\end{array}$ & Trivalent & 189 & $5 \cdot 7$ & $9 / 189$ & 4.8 & $4 / 189$ & 2.1 \\
\hline $\begin{array}{l}\text { Influvac } \\
\text { (BGP Products) }\end{array}$ & Trivalent & 47 & 1.4 & $5 / 47$ & NR & $2 / 47$ & $N R$ \\
\hline $\begin{array}{l}\text { Agrippal } \\
\text { (Novartis Vaccines and } \\
\text { Diagnostics) }\end{array}$ & Trivalent & 11 & 0.3 & $0 / 11$ & $\mathrm{NR}$ & $0 / 11$ & NR \\
\hline $\begin{array}{l}\text { FluQuadri } \\
\text { (Sanofi Pasteur) }\end{array}$ & Quadrivalent & 14 & 0.4 & $1 / 14$ & NR & $1 / 14$ & NR \\
\hline
\end{tabular}

NR: not relevant.

a Brand unknown for four participants.

${ }^{b}$ All administered vaccines except for FluQuadri were trivalent. Quadrivalent vaccines (FluQuadri/ FluQuadri Junior and Fluarix Tetra (GlaxoSmithKline)) were available for use for the first time in Australia in 2015 but were not funded under the National Immunisation Program.

${ }^{c} p=0.775$ for rates of fever among those who received Vaxigrip ( $\left.4.3 \%\right)$ compared with those who received Fluarix ( $\left.4.8 \%\right)$ calculated using Pearson's chi-square test.

${ }^{\mathrm{d}} \mathrm{p}=0.102$ for rates of medical advice/attendance sought among those who received Vaxigrip (o.9\%) compared with those who received Fluarix (2.1\%) calculated using Fisher's exact test.

seasonal influenza vaccines in children, AusVaxSafety surveillance data have been able to provide reassuring results.

Data obtained from parental reporting should be interpreted with care. Consequently, AusVaxSafety reports on outcomes which are the most objective: fever and medical advice/attendance sought within three days of vaccination. Although these provide less precision than results obtained in more formal follow-up such as clinical trials, this is unlikely to reduce our system's sensitivity for detecting SAEs, of which medical advice/ attendance sought can be considered a good proxy. This was demonstrated in the epidemiological investigation of the 2010 increase in febrile reactions [16].

An advantage of our system is its potential adaptability for monitoring new vaccines, such as live attenuated influenza vaccine, although this is not yet available in the southern hemisphere. Another advantage is its ability to provide rapid real-time feedback to inform programme rollout and vaccine promotion. In addition, AusVaxSafety's flexibility may be valuable in situations where vaccine safety data are limited, such as for pandemic vaccines. The timeliness of our results also makes them valuable beyond Australia; our data may be of interest to counterparts in the northern hemisphere preparing for 2015/16 vaccination using vaccines comprised of the strains administered in the 2015 southern hemisphere season.

Our system, which is able to report adverse events within days of vaccination, is as near to real time as possible. Such timeliness is feasible thanks to the strong collaboration with parents/carers and providers and the use of SMS technology for reporting reactions. We anticipate being able improve our system by including more participants in future years. To our knowledge, AusVaxSafety is the only active influenza vaccine safety surveillance system for young children analysing and reporting data on a weekly basis, allowing safety deliberations on vaccines within mere weeks of influenza vaccination commencing. Our ability to provide early and reliable safety profiles of seasonal influenza vaccines for children is likely to improve public confidence and vaccine uptake, which we will continue to assess.

\section{AusVaxSafety 2015 surveillance team}

Karen Orr, Gulam Khandaker, Kevin Yin, David Durrheim, Craig Dalton, Sally Munnoch, Michelle Butler, Jody Stephenson, Stephen Clarke, Keira Glasgow, Lauren Dalton, Brendan McMullan, Geraldine Dunne, Jim Buttery, Gowri Selvaraj, Annette Alafaci, Peter Eizenberg, Paul Effler, Peter Richmond, Peter Jacoby, Parveen Fathima, Lauren Tracey, Gabriela Willis, Jennifer Kent, Ian Peters, Rachel West, Kari Jarvinen, Susan Vlack, Deborah Judd, Melinda Hassall, Julia Clark, Stephen Lambert, Michael Gold, Gabriella Lincoln, Rosalind Webby, Kaylene Prince.

\section{Acknowledgements}

AusVaxSafety surveillance was funded under a contract with the Australian Government Department of Health. We would like to thank the AusVaxSafety Steering Committee members for their contribution to oversight of the 2015 surveillance effort. We would also like to express our gratitude to the staff 
at our participating hospitals, clinics and general practices, as well as the parents/carers of children who participated in and supported 2015 AusVaxSafety surveillance.

\section{Conflict of interest}

None declared.

\section{Authors' contributions}

AP served as AusVaxSafety surveillance coordinator for 2015, drafted the manuscript, and conducted data analysis and interpretation of results. PC contributed to the design and implementation of the AusVaxSafety surveillance system, served as coordinator/recruiter of the Hunter New England area surveillance sites, reviewed and contributed to the manuscript draft. AL contributed to the design and implementation of the AusVaxSafety surveillance system, recruited general practice site participants, reviewed and contributed to the manuscript draft. AR contributed to the design and implementation of the AusVaxSafety surveillance system, served as a coordinator of the Western Australia surveillance sites, conducted data collection and analysis, and reviewed and contributed to the manuscript draft. DW served as a coordinator of the Western Australia surveillance sites, conducted data collection and analysis, and reviewed and contributed to the manuscript draft. TS contributed to the design and implementation of the AusVaxSafety surveillance system, conducted data analysis, and reviewed the manuscript draft. CB contributed to the design and implementation of the AusVaxSafety surveillance system and reviewed and contributed to the draft manuscript. NC contributed to the design and implementation of the AusVaxSafety surveillance system and reviewed and contributed to the draft manuscript. KM contributed to the design, implementation and coordination of the AusVaxSafety surveillance system, and drafted the manuscript.

\section{References}

1. AusVaxSafety. AusVaxSafety Final report to the Australian Government Department of Health for Contract HEALTH/082/2014. Sydney: National Centre for Immunisation Research and Surveillance of Vaccine Preventable Diseases; Dec 2014.

2. Khandaker G. Going national: AusVaxSafety 2014 and beyond. Active surveillance for adverse events following immunisation - new methods in vaccine pharmacovigilance. Conference on Vaccine safety: active surveillance for adverse events following immunisation - new methods in vaccine pharmacovigilance; 29 Oct 2014; North Sydney, Australia.

3. Hull B, Dey A, Beard F, Menzies R, Brotherton J, Mclntyre $P$. Annual immunisation coverage report 2013. Westmead: National Centre for Immunisation Research and Surveillance of Vaccine Preventable Diseases; 2013. Available from: http:// ncirs.edu.au/assets/surveillance/coverage/2013-coveragereport-final.pdf

4. Australian demographic statistics, Mar 2015. Sydney: Australian Bureau of Statistics. [Accessed: 30 September 2015]. Available from: http://www.abs.gov.au/ausstats/abs@. $\mathrm{nsf} / \mathrm{mf} / 3101.0$

5. CashmanP, MoberleyS, DaltonC, StephensonJ, ElvidgeE, ButlerM, et al. Vaxtracker: Active on-line surveillance for adverse events following inactivated influenza vaccine in children. Vaccine. 2014;32(42):5503-8. DOI: 10.1016/j. vaccine.2014.07.061 PMID: 25077424

6. LeebA, ReganAK, PetersIJ, LeebC, LeebG, EfflerPV. Using automated text messages to monitor adverse events following immunisation in general practice. Med J Aust. 2014;200(7):4168. DOI: $10.5694 /$ mja13.11166 PMID: 24794676

7. MahajanD, DeyA, CookJ, HarveyB, MenziesRI, MacartneyKM. Surveillance of adverse events following immunisation in Australia, 2012.Commun Dis Intell Q Rep. 2014;38(3):E232-46. PMID: 25391415
8. Lucas], CrosierR. Fast initial response for cusum qualitycontrol schemes: give your cusum a head start.Technometrics. 1982;24(3):199-205. DOI: 10.1080/00401706.1982.10487759

9. GriggOA, FarewellVT, SpiegelhalterDJ. Use of risk-adjusted CUSUM and RSPRT charts for monitoring in medical contexts. Stat Methods Med Res. 2003;12(2):147-70.PMID: 12665208

10. BlythCC, MarkusTY, EfflerPV, RichmondPC. Ensuring safety of the 2011 trivalent influenza vaccine in young children. Med J Aust. 2011;195(1):52.PMID: 21728948

11. WoodNJ, BlythCC, WillisGA, RichmondP, GoldMS, ButteryJP, et al. The safety of seasonal influenza vaccines in Australian children in 2013. Med J Aust. 2014;201(10):596-600. DOI: 10.5694/mja13.00097 PMID: 25390267

12. Li-Kim-MoyJ, YinJK, RashidH, KhandakerG, KingC, WoodN, et al. Systematic review of fever, febrile convulsions and serious adverse events following administration of inactivated trivalent influenza vaccines in children. Euro Surveill. 2015;20(24):21159. DOI: 10.2807/1560-7917. ES2015.20.24.21159 PMID: 26111238

13. TraceyLE, ReganAK, MakDB, EfflerPV. Adverse events following influenza immunization reported by healthcare personnel using active surveillance based on text messages.Infect Control Hosp Epidemiol. 2015;36(5):608-10. DOI: 10.1017/ ice.2015.16 PMID: 25652211

14. ReganAK, BlythCC, MakDB, RichmondPC, EfflerPV. Using SMS to monitor adverse events following trivalent influenza vaccination in pregnant women. Aust N Z J Obstet Gynaecol. 2014;54(6):522-8. DOI: 10.1111/ajo.12266 PMID: 25306915

15. StockwellMS, BroderK, LaRussaP, LewisP, FernandezN, SharmaD, et al. Risk of fever after pediatric trivalent inactivated influenza vaccine and 13-valent pneumococcal conjugate vaccine. JAMA Pediatr. 2014;168(3):211-9. DOI: 10.1001/jamapediatrics.2013.4469 PMID: 24395025

16. ArmstrongPK, DowseGK, EfflerPV, CarcioneD, BlythCC, RichmondPC, et al. Epidemiological study of severe febrile reactions in young children in Western Australia caused by a 2010 trivalent inactivated influenza vaccine. BMJ Open. 2011;1(1):e000016. DOI: 10.1136/bmjopen-2010-000016 PMID: 22021725

17. RockmanS, DysonA, KoernigS, BecherD, NgM, MorelliAB, et al. Evaluation of the bioactivity of influenza vaccine strains in vitro suggests that the introduction of new strains in the 2010 Southern Hemisphere trivalent influenza vaccine is associated with adverse events. Vaccine. 2014;32(30):3861-8. DOI: 10.1016/j.vaccine.2014.03.032 PMID: 24928062

18. BlythCC, RichmondPC, JacobyP, ThorntonP, ReganA, RobinsC, et al. The impact of pandemic $A\left(\mathrm{H}_{1} \mathrm{~N}_{1}\right)$ pdmog influenza and vaccine-associated adverse events on parental attitudes and influenza vaccine uptake in young children. Vaccine. 2014;32(32):4075-81. DOI: 10.1016/j.vaccine.2014.05.055 PMID: 24877764

19. Government of Western Australia Department of Health. Child influenza vaccination rate low despite rise in uptake. Disease WAtch. 2013;17(4). Available from: http://www.health.wa.gov. au/diseasewatch/vol17_issue4/child_vaccination.cfm 